ESAIM: PROCEEDINGS AND SURVEYS, December 2014, Vol. 47, p. 1-16

F. Abergel, M. Aiguier, D. Challet, P.-H. Cournède, G. Faÿ, P. Lafitte, Editors

\title{
EXPLICIT EQUILIBRIA IN BILINEAR KINETIC MODELS FOR SOCIO-ECONOMIC INTERACTIONS
}

\author{
Federico Bassetti And Giuseppe Toscani ${ }^{1}$
}

\begin{abstract}
Bilinear kinetic models of Maxwell-Boltzmann type are often used to model socio-economic systems composed by agents that undergo binary interactions, which in general obey to some conservation law. Then, the details of the microscopic interaction are such that an equilibrium solution emerges. At difference with the classical Boltzmann equation for elastic gas particles, the analytic form of the equilibria is known only in some particular case. In the present note, we present and discuss the situations in which this equilibrium profile can be explicitly found. Applications to dissipative gases and to wealth redistribution models are presented.
\end{abstract}

Résumé. Des modèles cinétiques bilinéaire de type Maxwell-Boltzmann sont souvent utilisés pour modéliser des systèmes socio-économiques composées d'agents soumis à des interactions binaires. Ces modèles obéissent en général à une loi de conservation. Les détails microscopique de l'interaction conduisent le système vers un profil d'équilibre (une solution stationnaire). À la différence de l'équation de Boltzmann classique pour les particules de gaz élastiques, la forme analytique de la solution stationnaire n'est connue que dans certains cas particuliers. Dans cette note, nous présentons et discutons des situations pour lesquelles ce profil d'équilibre peut être trouvé explicitement. Des applications à des gaz dissipatifs et à des modèles de redistribution de richesse sont présentées.

\section{INTRODUCTION}

In the last two decades, kinetic theory of rarefied gases has been successfully applied to model socio-economic systems composed by a huge number of agents [27]. The main assumption which enlightens strong analogies with the classical kinetic theory of rarefied gases is that agents mostly interact by means of binary interactions. Maybe the most studied models are those relative to wealth distribution in a western society [27], where the principles of statistical mechanics have been fruitfully employed and led to the flowering of econophysics.

The prototype of these bilinear models can be easily introduced by resorting to the analogous description valid for a system of elastic molecules of an ideal gas. The basic assumption used to describe the various models for a spatially homogeneous gas, in which particles move only in one spatial direction, is that particles change their velocities only because of binary collisions. The most general (linear) binary interaction among two selected particles, can be described by assuming that their velocities, say $v$ and $w$, change to

$$
v^{\prime}=p_{1} v+q_{1} w \quad w^{\prime}=q_{2} v+p_{2} w
$$

\footnotetext{
${ }^{1}$ Department of Mathematics, University of Pavia, via Ferrata 1, Pavia, Italy.
} 
ESAIM: PROCEEDINGS AND SURVEYS

where $\left(p_{1}, q_{1}\right)$ and $\left(q_{2}, p_{2}\right)$ are two random vectors. In $(1)$ we denoted with $p_{i}, i=1,2$ (respectively $\left.q_{i}, i=1,2\right)$ the percentage of the pre-collision velocity which remains in the corresponding (respectively the other) postcollision velocity. The binary interaction (1) satisfies in general some conservation law. A typical one is the conservation of momentum, which asserts that

$$
v^{\prime}+w^{\prime}=v+w
$$

As mentioned before, one main example of this collision rule is given by the study of wealth distribution. In this case, the (non-negative) variables $v$ and $w$ have the meaning of the wealth of the two selected agents, and the collision (1) describes the details of the binary trade between agents. Hence (1) describes trades in which agents change their wealth according to some well-defined universal strategy. In this case, conservation of momentum in (2) simply means that the pair of agents exit from the trade with the same total amount of wealth they possessed before trading.

Given a binary collision rule of type (1) one can build, following the standard tools of kinetic theory, the corresponding kinetic equation for the particle density in the limit of infinite particles [27]. These bilinear kinetic equations can be described in the following abstract way. Set

$$
(L, R)=\left(p_{1}, q_{1}\right) \delta+\left(q_{2}, p_{2}\right)(1-\delta)
$$

where $\delta$ is a random variable stochastically independent of $p_{i}, q_{i}, i=1,2$, taking only the values 0,1 with equal probabilities and define, for every couple of probability measures $\left(\mu_{1}, \mu_{2}\right)$, the randomly weighed convolution

$$
Q^{+}\left(\mu_{1}, \mu_{2}\right)=\operatorname{Law}\left(L V_{1}+R V_{2}\right)
$$

where $V_{1}, V_{2},(L, R)$ are stochastically independent, and $V_{i}, i=1,2$, has law $\mu_{i}$. Then, the (weak form of the) bilinear kinetic equation corresponding to (1) reads

$$
\begin{aligned}
& \frac{d}{d t} \int_{\mathbb{R}} \varphi(x) \mu(t, d x)+\int_{\mathbb{R}} \varphi(x) \mu(t, d x)=\int_{\mathbb{R}} \varphi(x) Q^{+}(\mu(t), \mu(t))(d x), \\
& \lim _{t \rightarrow 0} \int_{\mathbb{R}} \varphi(x) \mu(t, d x)=\int_{\mathbb{R}} \varphi(x) \mu_{0}(d x)
\end{aligned}
$$

for any smooth function $\varphi(x)$. Here the solution $\mu(t)$ is a time-dependent probability measure on the real line $\mathbb{R}$ which describes the particle density at time $t$ and $\mu_{0}$ is the initial distribution of the particle.

Equation (4) can be equivalently and more conveniently written in Fourier variables as

$$
\partial_{t} \hat{\mu}(t, \xi)+\hat{\mu}(t, \xi)=\mathbb{E}[\hat{\mu}(t, L \xi) \hat{\mu}(t, R \xi)] \quad(\xi \in \mathbb{R})
$$

where $\hat{\mu}(t, \xi):=\int_{\mathbb{R}} e^{i \xi v} \mu(t)(d v)$ denotes the Fourier-Stieltjes transform of $\mu(v, t)$ and $\mathbb{E}$ denotes the expectation with respect to the joint law of $(L, R)$.

In this paper, we will be mainly concerned with the study of (explicit) steady states solutions to equation (4), or, alternatively, to its Fourier version (5). A probability distribution $\mu_{\infty}$ is a steady state for (4) if it satisfies

$$
\mu_{\infty}=Q^{+}\left(\mu_{\infty}, \mu_{\infty}\right)
$$

Note that equation (6) in terms of random variables reads

$$
X \stackrel{d}{=} L X_{1}+R X_{2}
$$

where $X, X_{1}, X_{2} \sim \mu_{\infty}, X_{1}, X_{2},(L, R)$ are stochastically independent and $\stackrel{d}{=}$ means equality in distribution. Here and after, the notation $X \sim \mu$ is a shorthand to say that the random variable $X$ is distributed with law $\mu$. 
Usually, the presence of suitable conservation laws - like (2) - yields the existence of a steady state. Then, the details of the microscopic interactions (1) induce certain properties at the macroscopic level, which can be read by looking at the steady state [27]. However, on the contrary to what happens in the classical kinetic theory of rarefied gases, where the stationary solution is explicitly known (the Maxwellian density), in the general case one can only reach few properties of the steady states, but not their analytic expressions, with the exception of few particular cases, see e.g. [8].

In this paper, we discuss in details some of the (relatively few) cases in which equilibria are explicitly known. These cases refer to bilinear kinetic models describing wealth distribution and dissipative gases.

Our results will take essential advantage from the linearity of the interactions, expressed by (1) (respectively (7)). Consequently, other kinetic models of interest for applications, in which the underlying interactions are nonlinear in terms of the pre-collision variables, fall out of our treatise. This is the case, for example, of the various kinetic models for opinion formation considered into the pertinent literature the last ten years, starting from [32] (cf. in particular [12,13]).

\section{REVIEW OF GENERAL RESUlts}

\subsection{Stable laws and domain of attraction}

We will start by briefly recalling some known facts about stable laws. A probability distribution $g_{\alpha}$ is said to be a centered stable law of exponent $\alpha$ (with $\alpha \in(0,2])$ and real parameters $(\lambda, \beta), \lambda>0$ and $|\beta| \leq 1$, if its Fourier-Stieltjes transform $\hat{g}_{\alpha}(\xi)=\int_{\mathbb{R}} e^{i \xi v} g_{\alpha}(d v)$ has the form

$$
\hat{g}_{\alpha}(\xi)= \begin{cases}\exp \left\{-\lambda|\xi|^{\alpha}(1-i \beta \tan (\pi \alpha / 2) \operatorname{sign} \xi)\right\} & \text { if } \alpha \in(0,1) \cup(1,2) \\ \exp \{-\lambda|\xi|(1+2 i \beta / \pi \log |\xi| \operatorname{sign} \xi)\} & \text { if } \alpha=1 \\ \exp \left\{-\lambda|\xi|^{2}\right\} & \text { if } \alpha=2 .\end{cases}
$$

By definition, a probability measure $\mu$ belongs to the normal domain of attraction (briefly NDA) of a stable law of exponent $\alpha$, if for any sequence of independent and identically distributed real-valued random variables $\left(X_{n}\right)_{n \geq 1}$ with common distribution $\mu$, there exists a sequence of real numbers $\left(c_{n}\right)_{n \geq 1}$ such that the law of $n^{-1 / \alpha} \sum_{i=1}^{n} X_{i}-c_{n}$ converges weakly to a stable law of exponent $\alpha$.

It is well-known that, provided $\alpha \neq 2$, a probability measure $\mu$ belongs to the NDA of an $\alpha$-stable law if and only if it shares with the stable law the decay at infinity, more precisely if and only if

$$
\lim _{x \rightarrow+\infty} x^{\alpha} \mu\{(x,+\infty)\}=c_{0}^{+}<+\infty, \quad \lim _{x \rightarrow-\infty}|x|^{\alpha} \mu\{(-\infty, x]\}=c_{0}^{-}<+\infty
$$

Typically, in order to exclude convergence to the probability measure concentrated at zero, one also requires that $c_{0}^{+}+c_{0}^{-}>0$. Here we shall also include the situation $c_{0}^{+}=c_{0}^{-}=0$ as a special case. The parameters $\lambda$ and $\beta$ of the associated stable law in (8) are related to $c_{0}^{+}$and $c_{0}^{-}$by the relations

$$
\lambda=\frac{\left(c_{0}^{+}+c_{0}^{-}\right) \pi}{2 \Gamma(\alpha) \sin (\pi \alpha / 2)}, \quad \beta=\frac{c_{0}^{+}-c_{0}^{-}}{c_{0}^{+}+c_{0}^{-}},
$$

with the convention that $\beta=0$ if $c_{0}^{+}+c_{0}^{-}=0$ [22]. Above, $\Gamma(\cdot)$ denotes as usual the Euler Gamma function. The case $\alpha=2$, i.e. the Gaussian case, is distinct from the others. The probability distribution $\mu$ belongs to the normal domain of attraction of a Gaussian law if and only if it has finite variance $\sigma^{2}$. The parameter $\lambda$ of the associated Gaussian law in (8) is now given by $\lambda=\sigma^{2} / 2$.

When $\alpha \in(0,1)$ and $\beta=1$, the corresponding stable distribution is supported on $\mathbb{R}^{+}$, so that $g_{\alpha}\left(\mathbb{R}^{+}\right)=1$. Analogously, when $\alpha \in(0,1)$ and $\beta=-1$ one has $g_{\alpha}\left(\mathbb{R}^{-}\right)=1$. In all the other cases, the stable distributions are supported on $\mathbb{R}$ [33]. In particular, if $\alpha \in(0,1)$ and $\beta=1$ the Laplace transform of an $\alpha$-stable law of 
parameters $(\lambda, \beta=1)$ is given by $[33]$

$$
\int e^{-\xi x} g_{\alpha}(d x)=\exp \left\{-\frac{\lambda}{\cos (\pi \alpha / 2)} \xi^{\alpha}\right\} \quad(\xi>0) .
$$

A well-known useful result, that goes back to Ibragimov and Linnik [22], gives a complete characterization of the normal domain of attraction of a probability distribution $\mu$ in term of its Fourier-Stieltjes transform $\hat{\mu}(\xi)=\int \exp (i \xi x) \mu(d x)$.

Theorem 2.1 ( $[1,22])$. Let $\alpha \in(0,2)$. Then $\mu$ satisfies (9) with $c_{0}^{+}+c_{0}^{-}>0$ if and only if, as $\xi \rightarrow 0$,

$$
\log (\hat{\mu}(\xi))=i \xi k_{0}+\log \left(\hat{g}_{\alpha}(\xi)\right)+o\left(|\xi|^{\alpha}\right)
$$

where $k_{0}$ is a constant and $\hat{g}_{\alpha}$ is given by (8). Analogously, when $\alpha=2, \mu$ has finite variance if and only if, as $\xi \rightarrow 0$

$$
\log (\hat{\mu}(\xi))=i \xi k_{0}-\frac{\sigma^{2}}{2}|\xi|^{2}+o\left(|\xi|^{2}\right)
$$

Moreover, for $\alpha>1, k_{0}=\int x \mu(d x)$.

\subsection{Convergence to steady states}

Given $\alpha \in(0,2]$ we introduce two important assumptions. The first one concerns the collision coefficients $(L, R)$, the second one the initial distribution $\mu_{0}$.

$\left(H_{0}\right) L$ and $R$ are non-negative random variables such that

$$
\mathbb{P}\{L>0\}+\mathbb{P}\{R>0\}>1, \quad \mathbb{E}\left[L^{\alpha}+R^{\alpha}\right]=1, \quad \text { and } \quad \mathbb{E}\left[L^{p}+R^{p}\right]<1
$$

for some $p>0$.

$\left(H_{\alpha}\right)$ The measure $\mu_{0}$ is such that:

- when $\alpha \in(0,1)$ then $\mu_{0}$ satisfies $(9)$;

- when $\alpha=1$ then $\mu_{0}$ satisfies (9) with $c_{0}^{-}=c_{0}^{+}$and

$$
\lim _{R \rightarrow+\infty} \int_{(-R, R)} x \mu_{0}(d x)=k_{0}
$$

with $-\infty<k_{0}<+\infty$;

- when $\alpha \in(1,2)$ then $\mu_{0}$ satisfies (9) and $\int x \mu_{0}(d x)=0$;

- when $\alpha=2$ then $\int x^{2} \mu_{0}(d x)=\sigma^{2}<+\infty$ and $\int x \mu_{0}(d x)=0$.

These assumptions allow to describe in a precise way the asymptotic behavior of equation (4).

Theorem 2.2 ( $[6])$. Assume that $\left(H_{0}\right)-\left(H_{\alpha}\right)$ hold true with $\alpha \neq 1$. If $p<\alpha, \mu_{t}$ converges weakly to the degenerate probability measure $\delta_{0}$. Conversely, if $p>\alpha, \mu_{t}$ converges weakly to a steady state $\mu_{\infty}$ with Fourier-Stieltjes transform

$$
\int_{\mathbb{R}} e^{i \xi x} \mu_{\infty}(d x)=\int_{\mathbb{R}^{+}} e^{-\lambda m|\xi|^{\alpha}\left[1-i \beta \tan \left(\alpha \frac{\pi}{2}\right) \operatorname{sign} \xi\right]} \nu_{\alpha}(d m) \quad(\xi \in \mathbb{R}) .
$$

In (12), $\nu_{\alpha}$ is a suitable probability measure on $\mathbb{R}^{+}=[0,+\infty)$, such that $\int_{\mathbb{R}^{+}} m \nu_{\alpha}(d m)=1$, and the parameters $\lambda$ and $\beta$ are defined in (10) for $\alpha<2$ and $(\lambda, \beta)=\left(\sigma^{2} / 2,0\right)$ for $\alpha=2$. 
Theorem $2.3([7])$. Assume that $\left(H_{0}\right)-\left(H_{\alpha}\right)$ hold with $\alpha=1$. If $p<1$, then $\mu_{t}$ converges weakly to the degenerate probability measure $\delta_{0}$. Conversely, if $p>1, \mu_{t}$ converges weakly, as $t \rightarrow+\infty$, to a steady state $\mu_{\infty}$ with Fourier-Stieltjes transform

$$
\int_{\mathbb{R}} e^{i \xi x} \mu_{\infty}(d x)=\int_{\mathbb{R}^{+}} e^{m\left(i k_{0} \xi-c_{0}^{+} \pi|\xi|\right)} \nu_{1}(d m)
$$

In $(13), \nu_{1}$ is a suitable probability measure on $\mathbb{R}$ such that $\int_{[0,+\infty)} m \nu_{1}(d m)=1$.

The distribution $\nu_{\alpha}$ appearing in the previous theorems can be characterized in a precise way: $\nu_{\alpha}$ is the unique probability distribution such that $\nu_{\alpha}\left(\mathbb{R}^{+}\right)=1, \int_{\mathbb{R}^{+}} x \nu_{\alpha}(d x)=1$ and

$$
\hat{\nu}_{\alpha}(\xi)=\mathbb{E}\left[\nu_{\alpha}\left(\xi L^{\alpha}\right) \nu_{\alpha}\left(\xi R^{\alpha}\right)\right] \quad(\xi \in \mathbb{R})
$$

Note that $\nu_{\alpha}$ does not depend on the initial condition $\mu_{0}$. Positive solutions of (14) (known as positive fixed points of the smoothing transformation) are deeply studied, in particular in connection with the so-called Branching Random Walk, see e.g. [2,17,23,25]. Moreover, it has been recently proven in [3] that the set of all the solutions of equation (6) coincides precisely with the mixtures of stable laws defined in (12) for $\alpha \neq 1$ and in (13) for $\alpha=1$, under the mild additional assumption that $P\left\{L, R \in\{0\} \cup r^{\mathbb{Z}}\right\}<1$ for all $r>1$. We lastly mention that self-similar solutions to equation (4) are studied in $[5,11]$.

\section{EXAMPLES OF EXPLICIT SOLUTIONS}

\subsection{Beta and Gamma distributions}

To find explicitly steady states to equation (4), we need some preliminary computations, which are mainly related to well-known special functions. We first recall formula 3.197.4 for integrals in [20]. This formula gives, for any $\alpha_{1}>0, \alpha_{2}>0, \xi>0$,

$$
\frac{\Gamma\left(\alpha_{1}+\alpha_{2}\right)}{\Gamma\left(\alpha_{1}\right) \Gamma\left(\alpha_{2}\right)} \int_{0}^{1} \frac{x^{\alpha_{1}-1}(1-x)^{\alpha_{2}-1}}{(1+\xi x)^{\alpha_{1}+\alpha_{2}}} d x=\frac{1}{(1+\xi)^{\alpha_{1}}} .
$$

Note that identity (15) is a special case of Euler's transformation for Hypergeometric functions. Indeed Euler's representation of the Hypergeometric function ${ }_{2} F_{1}(a, b ; c ; z)$ is

$$
{ }_{2} F_{1}(a, b ; c ; z)=\frac{\Gamma(b)}{\Gamma(b) \Gamma(c-b)} \int_{0}^{1} \frac{x^{b-1}(1-x)^{c-b-1}}{(1-z x)^{a}} d x
$$

for $\operatorname{Re}(c)>\operatorname{Re}(b)>0$ and $|z|<1$. The previous formula gives also the analytic continuation of ${ }_{2} F_{1}(a, b ; c ; z)$ for any complex $z$ with $|\arg (1-z)|<\pi$ (cf. formula 2.1.3 of [18]). Now formula 2.1.4 of [18] shows that Euler's transformation is given by

$$
{ }_{2} F_{1}(a, b ; c ; z)=\frac{1}{(1-z)^{a+b-c}}{ }_{2} F_{1}(c-a, c-b ; c ; z)
$$

for $|z|<1$. When $a=c=\alpha_{1}+\alpha_{2}, b=\alpha_{1}$ and $z=-\xi$ this last equation reduces to (15) for $0<\xi<1$. Analytic continuation gives (15) for $\xi>0$.

A random variable $B$ is said to have a Beta distribution of parameters $(a, b)$ (in symbols $B \sim B e t a(a, b)$ ), if its density is given by

$$
x \mapsto \frac{\Gamma(a+b)}{\Gamma(a) \Gamma(b)} x^{a-1}(1-x)^{b-1} \mathbb{I}_{(0,1)}(x),
$$


for $a>0, b>0$. We denoted by $\mathbb{I}_{A}(x)$ the indicator function of a set $A$. Note that the uniform distribution on $(0,1)$ is obtained for $a=b=1$.

A random variable $G$ is said to have a Gamma distribution of parameter $(\gamma, \theta)$ (in symbols $G \sim \operatorname{Gamma}(\gamma, \theta)$ ), if its density is given by

$$
x \mapsto \frac{x^{\gamma-1} e^{-x / \theta}}{\Gamma(\gamma) \theta^{\gamma}} \mathbb{I}_{(0,+\infty)}(x)
$$

with $\theta>0$ and $\gamma>0$, or, analogously, if its Laplace transform, for $\xi>0$, is given by

$$
\mathbb{E}\left[e^{-\xi G}\right]=\frac{1}{(1+\theta \xi)^{\gamma}} .
$$

Note that in this parametrization $\theta$ is a scale parameter. Clearly the exponential distribution is obtained from (17) as the special case in which $\gamma=1$.

As a first application of (15), by computing Laplace transforms one can reckon that if $\operatorname{G\sim Gamma(\gamma ,\theta )}$ and $B \sim \operatorname{Bet} a(a, b)$ are independent with $a+b=\gamma$, then

$$
B \cdot G \sim \operatorname{Gamma}(a, \theta)
$$

Hence, in particular,

$$
G_{1} \stackrel{d}{=} B\left(G_{1}+G_{2}\right)
$$

where $B \sim \operatorname{Beta}(a, a)$ and $G_{i} \sim \operatorname{Gamma}(a, \theta), i=1,2$, are independent. This example can be easily extended to more general distributions, known as Generalized Linnik distributions.

\subsection{Generalized Linnik distributions are steady states}

A random variable $X$ with characteristic function $[15,24]$

$$
\mathbb{E}\left[e^{i \xi X}\right]=\frac{1}{\left(1+\theta|\xi|^{\alpha}\right)^{\gamma}}, \quad \xi \in \mathbb{R},
$$

where $\theta>0, \gamma>0$, and $\alpha \in(0,2]$, is said to have (generalized) symmetric Linnik law of parameters $(\alpha, \gamma, \theta)$ (in symbols $\operatorname{SLi}(\alpha, \gamma, \theta))$.

A positive random variable $X$ is said to have positive Linnik distribution (in symbols $P \operatorname{Li}(\alpha, \gamma, \theta)$ ), if its Laplace transform is given by

$$
\mathbb{E}\left[e^{-\xi X}\right]=\frac{1}{\left(1+\theta \xi^{\alpha}\right)^{\gamma}}, \quad \xi \in \mathbb{R}^{+},
$$

where $\theta>0, \gamma>0$ and $\alpha \in(0,1]$. Note that in this second case the parameter $\alpha$ belongs to a reduced interval $\alpha \in(0,1]$. Note also that $P \operatorname{Li}(1, \gamma, \theta)$ is nothing but the Gamma distribution of parameters $(\gamma, \theta)$. By using Lemma A.2 it is immediate to reckon that (19) and (19) are Fourier transform and Laplace transform (respectively). More precisely, $\operatorname{PLi}(\alpha, \gamma, \theta)$ and $\operatorname{SLi}(\alpha, \gamma, \theta)$ are stable mixture of gamma distributions.

Let now $\alpha \in(0,2], \gamma>0$ and $\theta>0$, and let us choose the random vector $(L, R):=\left(B^{1 / \alpha}, B^{1 / \alpha}\right)$, with $B \sim \operatorname{Beta}(\gamma, \gamma)$ and $X, X_{1}, X_{2} \sim \operatorname{SLi}(\alpha, \gamma, \theta)$. If $X_{1}, X_{2}, B$ are independent, combining formulas (15) and (19) one obtains

$$
\begin{aligned}
\mathbb{E}\left[e^{i \xi\left(L X_{1}+R X_{2}\right)}\right]=\mathbb{E}\left[\frac{1}{\left(1+\theta B|\xi|^{\alpha}\right)^{2 \gamma}}\right] & =\frac{\Gamma(2 a)}{\Gamma(a)^{2}} \int_{0}^{1} \frac{x^{\gamma-1}(1-x)^{a-1}}{\left(1+\theta|\xi|^{\alpha} x\right)^{2 \gamma}} d x \\
& =\frac{1}{\left(1+\theta|\xi|^{\alpha}\right)^{\gamma}}=\mathbb{E}\left[e^{i \xi X}\right] .
\end{aligned}
$$

This shows that, with the previous choices, (7) holds. Similar computations can be done by choosing $L^{\alpha} \sim$ $\operatorname{Beta}\left(a_{1}, a_{2}\right), R^{\alpha} \sim \operatorname{Beta}\left(a_{2}, a_{1}\right), \gamma=a_{1}+a_{2}$ with $L$ and $R$ stochastically independent. Another example 
of the same kind can be obtained by taking $(L, R)=\left(B_{1}^{1 / \alpha}, B_{1}^{1 / \alpha} B_{2}^{1 / \alpha}\right)$ where $B_{1}$ and $B_{2}$ are stochastically independent, $B_{1} \sim \operatorname{Bet} a\left(\gamma, a_{1}\right)$ and $B_{2} \sim \operatorname{Beta}\left(a_{1}, a_{2}\right)$ with $a_{1}+a_{2}=\gamma$.

Matching these considerations with the results of Theorems 2.2 and 2.3 one obtains the following:

Proposition 3.1. Let $\left(H_{\alpha}\right)$ hold with $c_{0}^{-}=c_{0}^{+}>0$ for some $\alpha \in(0,2]$. If $\alpha=1$ let us assume in addition that $k_{0}=0$. Lastly, let us assume that for some $\gamma>0, a_{1}>0, a_{2}>0$ one of the following conditions holds

(a) $(L, R)=\left(B_{0}^{1 / \alpha}, B_{0}^{1 / \alpha}\right)$, with $B_{0} \sim \operatorname{Beta}(\gamma, \gamma)$.

(b) $(L, R)=\left(B_{1}^{1 / \alpha}, B_{2}^{1 / \alpha}\right)$, with $B_{1}$ and $B_{2}$ stochastically independent, $B_{1} \sim \operatorname{Beta}\left(a_{1}, a_{2}\right), B_{2} \sim \operatorname{Beta}\left(a_{2}, a_{1}\right)$ and $a_{1}+a_{2}=\gamma$.

(c) $(L, R)=\left(B_{1}^{1 / \alpha}, B_{1}^{1 / \alpha} B_{2}^{1 / \alpha}\right)$ with $B_{1}$ and $B_{2}$ stochastically independent, $B_{1} \sim \operatorname{Beta}\left(\gamma, a_{1}\right), B_{2} \sim$ $\operatorname{Beta}\left(a_{1}, a_{2}\right)$ and $a_{1}+a_{2}=\gamma$.

Then, $\mu(t)$ converges weakly to a stationary state $\mu_{\infty}$ which is a (generalized) symmetric Linnik law of parameters $(\alpha, \gamma, \theta)$

$$
\hat{\mu}_{\infty}(\xi)=\frac{1}{\left(1+\theta|\xi|^{\alpha}\right)^{\gamma}},
$$

where $\theta=c_{0}^{+} \pi(\gamma \Gamma(\alpha) \sin (\pi \alpha / 2))^{-1}$ when $\alpha \neq 2$ and $\theta=\sigma^{2} /(2 \gamma)$ when $\alpha=2$.

Proof. Note that, if hypothesis (a) holds,

$$
\mathbb{E}\left[L^{\alpha}+R^{\alpha}\right]=2 \mathbb{E}[B]=1, \quad \mathbb{E}\left[L^{2 \alpha}+R^{2 \alpha}\right]=2 \mathbb{E}\left[B^{2}\right]=\frac{\gamma+1}{2 \gamma+1}<1
$$

and the random vector $(L, R)$ satisfies assumption $\left(H_{0}\right)$.

Whenever hypothesis (b) is given,

$$
\mathbb{E}\left[L^{2 \alpha}+R^{2 \alpha}\right]=\frac{a_{1}\left(a_{1}+1\right)+a_{2}\left(a_{2}+1\right)}{\gamma(\gamma+1)}<1
$$

and the random vector $(L, R)$ still satisfies assumption $\left(H_{0}\right)$. Similar computations can be done in case $(\mathrm{c})$ to prove (11). Thanks to Theorems 2.2 and 2.3 we conclude that the solution $\mu(t)$ to equation (4) converges weakly to a steady state. Since we have shown that $\mu_{\infty}$ given in (21) is a steady state, it remains to determine the correct value of $\theta$. This can be easily deduced by Theorem 2.1. Indeed

$$
\log \left(\hat{\mu}_{\infty}(\xi)\right)=\gamma \theta|\xi|^{\alpha}+o\left(|\xi|^{\alpha}\right)
$$

Hence, for $\alpha<2, \mu_{\infty}$ attracts all the initial conditions $\mu_{0}$ satisfying $\left(H_{\alpha}\right)$ with $c_{0}^{+}=c_{0}^{-}$and $c_{0}^{+}=\theta \gamma \Gamma(\alpha) \sin (\pi \alpha / 2) / \pi$. Analogous considerations can be done for $\alpha=2$.

Whenever $\alpha \leq 1$, a similar result can be obtained for $X, X_{1}, X_{2} \sim P \operatorname{Li}(\alpha, \gamma, \theta)$, using the Laplace transform in place of the characteristic function. The details are left to the reader.

Proposition 3.2. Assume that $\left(H_{\alpha}\right)$ hold for some $\alpha \in(0,1]$ with $c_{0}^{-}=0$. Assume in addition that $c_{0}^{+}=0$ and $k_{0}>0$ if $\alpha=1$. Finally assume that one of the conditions $(a),(b)$ or $(c)$ of Proposition 3.1 holds for some $\gamma>0, a_{1}>0, a_{2}>0$. Then, $\mu(t)$ converges weakly to a stationary state $\mu_{\infty}$ which is a positive Linnik distribution

$$
\int_{[0,+\infty)} e^{-\xi v} \mu_{\infty}(d v)=\frac{1}{\left(1+\theta \xi^{\alpha}\right) \gamma},
$$

where $\theta=\left(c_{0}^{+}\right) \pi(2 \gamma \Gamma(\alpha) \sin (\pi \alpha / 2) \cos (\pi \alpha / 2))^{-1}$ if $\alpha<1$ and $\theta=k_{0} / \gamma$ if $\alpha=1$.

Proposition 3.2, case (a), contains the results obtained in [8] about the explicit solutions for the pure gambling model, as it will be discussed in Section 4.1. 


\subsection{Inverse gamma distributions are steady states}

The examples of this Subsection are re-elaborations of [8]. A random variable $X$ is said to have inverse Gamma distribution of parameters $(\theta, \gamma)$ (in symbols $X \sim \operatorname{Inv} \operatorname{Gamma}(\gamma, \theta)$ ), if its density reads

$$
x \mapsto \frac{\theta^{\gamma}}{\Gamma(\gamma)} \frac{1}{x^{\gamma+1}} e^{-\theta / x} \mathbb{I}_{(0,+\infty)}(x) .
$$

It is well known and easily shown that if $G \sim \operatorname{Gamma}(\gamma, 1 / \theta)$ then $G^{-1} \sim \operatorname{Inv} \operatorname{Gamma}(\gamma, \theta)$. Moreover, the Laplace transform of an $\operatorname{Inv} \operatorname{Gamma}(\gamma, \theta)$ random variable is

$$
\mathbb{E}\left[e^{-\xi X}\right]=\frac{2(\theta \xi)^{\frac{\gamma}{2}} K_{\gamma}(2 \sqrt{\theta \xi})}{\Gamma(\gamma)}, \quad \xi \in \mathbb{R}^{+},
$$

where $K_{\gamma}$ is the Modified Bessel function of second kind [20]. Equation (24) follows by the well-known integral representation of $K_{\gamma}$

$$
K_{\gamma}(z)=\frac{1}{2}\left(\frac{z}{2}\right)^{\gamma} \int_{0}^{+\infty} \frac{1}{x^{\gamma+1}} e^{-x-z^{2} /(4 x)} d x .
$$

See, e.g., 8.432.6 [20]. Note that the characteristic function of $X$ is

$$
\xi \mapsto \mathbb{E}\left[e^{i \xi X}\right]=\frac{2(-i \theta \xi)^{\frac{\gamma}{2}} K_{\gamma}(2 \sqrt{-i \theta \xi})}{\Gamma(\gamma)} .
$$

At this stage, we need the recall a well-known fact on product of beta random variables: if $B_{1} \sim \operatorname{Beta}(a, b)$ and $B_{2} \sim \operatorname{Beta}(a+b, c)$ are independent, then

$$
B_{1} B_{2} \sim \operatorname{Beta}(a, b+c) .
$$

See e.g. [29].

Lemma 3.3. Let $\gamma>1 / 2$. Let $X_{0} \sim \operatorname{InvGamma}(2 \gamma, \theta), X_{1}, X_{2} \sim \operatorname{InvGamma}(\gamma, \theta), B_{0} \sim \operatorname{Beta}(\gamma, 1 / 2)$, $B_{1} \sim \operatorname{Beta}(\gamma+1 / 2, \gamma-1 / 2)$ with $X_{0}, X_{1}, X_{2}, B_{0}, B_{1}$ independent. Then the following are true:

$$
\begin{aligned}
& X_{1}+X_{2} \stackrel{d}{=} \frac{4 X_{0}}{B_{0}}, \\
& X_{1} \stackrel{d}{=} \frac{X_{1}+X_{2}}{4 B_{1}} .
\end{aligned}
$$

Moreover, $4 X_{0} / B_{0}$ has density

$$
\frac{\theta^{\gamma} 2^{2 \gamma} \Gamma(\gamma+1 / 2)}{\Gamma(\gamma)^{2} \sqrt{\pi}} x^{-\gamma-1} e^{-\frac{2 \theta}{x}} K_{0}\left(\frac{2 \theta}{x}\right) \mathbb{I}_{(0,+\infty)}(x),
$$

and $(0, \gamma+1 / 2) \ni s \mapsto g(s):=2 \mathbb{E}\left[\left(4 B_{1}\right)^{-s}\right]<+\infty$ is a convex function such that $g(\gamma)=g(1)=1$.

Proof. Since $\theta$ is a scale parameter, it clearly suffices to prove the statements for $\theta=1$. Let us first prove (26). Formula 6.592 .8 in [20], after a simple change of variables, gives

$$
K_{\gamma}(a)^{2}=\int_{0}^{1} u^{-1}(1-u)^{-1 / 2} K_{2 \gamma}\left(\frac{2 a}{\sqrt{u}}\right) d u \quad(a>0) .
$$


Now let $L_{\gamma}(\xi)=\mathbb{E}\left[e^{-\xi X_{1}}\right]$ and $L_{2 \gamma}=\mathbb{E}\left[e^{-\xi X_{0}}\right]$, recalling that we are assuming $\theta=1$, a combination of (24) and (29) gives

$$
\begin{aligned}
\mathbb{E}\left[e^{-\xi\left(X_{1}+X_{2}\right)}\right] & =L_{\gamma}(\xi)^{2}=\frac{4 \xi^{\gamma}}{\Gamma(\gamma)^{2}} K_{\gamma}^{2}(2 \sqrt{\xi})=\frac{4 \xi^{\gamma}}{\Gamma(\gamma)^{2}} \int_{0}^{1} u^{-1}(1-u)^{-1 / 2} K_{2 \gamma}\left(\frac{2 \sqrt{4 \xi}}{\sqrt{u}}\right) d u \\
& =\frac{2 \Gamma(2 \gamma)}{2^{2 \gamma} \Gamma(\gamma)^{2}} \int_{0}^{1} u^{\gamma-1}(1-u)^{-1 / 2} L_{2 \gamma}\left(\frac{4 \xi}{u}\right) d u
\end{aligned}
$$

Using the duplication formula $\Gamma(2 \gamma)=2^{2 \gamma-1} \Gamma(\gamma) \Gamma(\gamma+1 / 2) / \Gamma(1 / 2)$ (see 8.335.1 in [20]) one obtains

$$
L_{\gamma}(\xi)^{2}=\frac{\Gamma(\gamma+1 / 2)}{\Gamma(\gamma) \Gamma(1 / 2)} \int_{0}^{1} u^{\gamma-1}(1-u)^{-1 / 2} L_{2 \gamma}\left(\frac{4 \xi}{u}\right) d u
$$

which gives (26). As for (28) it follows by writing the density of $4 X_{0} / B_{0}$ and using the relation

$$
\int_{0}^{1} x^{-1}(1-x)^{-1 / 2} e^{-a / x} d x=e^{-a / 2} K_{0}(a / 2) \quad(a>0) .
$$

See, e.g., 3.471 .4 in [20]. Now let $X_{1}, X_{2} \sim \operatorname{Inv} \operatorname{Gamma}(\gamma, 1), X_{0} \sim \operatorname{Inv} \operatorname{Gamma}(2 \gamma, 1), Y_{0} \sim \operatorname{Gamma}(2 \gamma, 1)$ $B_{0} \sim \operatorname{Beta}(\gamma, 1 / 2)$ and $B_{1} \sim \operatorname{Beta}(\gamma+1 / 2, a-1 / 2)$, then by (26) one has

$$
\frac{X_{1}+X_{2}}{4 B_{1}} \stackrel{d}{=} \frac{X_{0}}{B_{0} B_{1}} \stackrel{d}{=} \frac{1}{B_{0} B_{1} Y_{0}} .
$$

Now by $(25)$ it follows that $B_{0} B_{1} \sim \operatorname{Beta}(\gamma, \gamma)$ and hence, by (18), $B_{0} B_{1} Y_{0} \sim \operatorname{Gamma}(\gamma, 1)$. Hence $\left(B_{0} B_{1} Y_{0}\right)^{-1} \sim$ $\operatorname{Inv} \operatorname{Gamma}(\gamma, 1)$. To conclude note that clearly $g(s)$ is convex and finite for $s \in(0, \gamma+1 / 2)$, moreover

$$
g(s)=\frac{2^{-2 s+1} \Gamma(\gamma-s+1 / 2) \Gamma(2 \gamma)}{\Gamma(2 \gamma-s) \Gamma(\gamma+1 / 2)}
$$

Recalling that $\Gamma(z+1) / \Gamma(z)=z$, one gets

$$
g(1)=\frac{1}{2} \frac{2 \gamma-1}{\gamma-1 / 2}=1
$$

While for $s=\gamma$

$$
g(\gamma)=2^{-2 \gamma+1} \frac{\Gamma(1 / 2) \Gamma(2 \gamma)}{\Gamma(\gamma) \Gamma(\gamma+1 / 2)}=1,
$$

where the last equality follows by the duplication formula recalled above. Note that a different proof of (27) is given in [8].

Proposition 3.4. Let $\gamma>1 / 2$ and $\gamma \neq 1$. Assume that $\left(H_{1}\right)$ holds with $c_{0}^{+}=c_{0}^{-}=0$ and $k_{0}>0$ if $\gamma>1$ and that $\left(H_{\gamma}\right)$ holds if $\gamma \in(1 / 2,1)$ with $c_{0}^{-}=0$.

(a) Let $(L, R)=\left(\left(4 B_{1}\right)^{-1},\left(4 B_{1}\right)^{-1}\right)$, with $B_{1} \sim \operatorname{Beta}(\gamma+1 / 2, \gamma-1 / 2)$. Then, $\mu(t)$ converges weakly to a stationary state $\mu_{\infty}$ which is InvGamma $(\gamma, \theta)$ for $\theta=k_{0}(\gamma-1)$ if $\gamma>1$ and $\theta=\left(c_{0}^{+} \gamma \Gamma(\gamma)\right)^{1 / \gamma}$ if $\gamma \in(1 / 2,1)$.

(b) Let $(L, R)=\left(\left(4 B_{1}\right)^{-1},\left(4 B_{2}\right)^{-1}\right)$, with $B_{i} \sim \operatorname{Beta}(\gamma+1 / 2, \gamma-1 / 2)$ stochastically independent. Then, $\mu(t)$ converges weakly to a stationary state $\mu_{\infty}$ with density given by (28) for $\theta=k_{0}(\gamma-1) / 2$ if $\gamma>1$ and $\theta=\left(c_{0}^{+} 2 \gamma \Gamma(\gamma)\right)^{1 / \gamma}$ if $\gamma \in(1 / 2,1)$. 
Proof of (a). We already know by $(27)$, that $\operatorname{Inv} \operatorname{Gamma}(\gamma, \theta)$ is a stationary state of $(4)-(3)$ for $(L, R)=$ $\left(\left(4 B_{1}\right)^{-1},\left(4 B_{1}\right)^{-1}\right)$. Assume first that $\gamma>1$. By the previous Lemma we know that $g$ is convex and $g(1)=$ $g(\gamma)=1$. This shows that $H_{0}$ is valid for $\alpha=1$ and $1<p<\gamma$. Hence Theorem 2.3 gives that $\mu(t)$ converges weakly to a stationary state. It remains to observe that, since for $\gamma>1$ the mean of an $\operatorname{Inv} \operatorname{Gamma}(\gamma, \theta)$ random variable is finite and equal to $\theta /(\gamma-1)$, it must be $\theta=k_{0}(\gamma-1)$. If $\gamma<1$, by the previous Lemma we know that $\left(H_{0}\right)$ is valid for $\alpha=\gamma$ and $\gamma<p<1$. Hence Theorem 2.2 gives that $\mu(t)$ converges weakly to a stationary state which is a mixture of $\gamma$-stable distribution. Using Lemma A.1 we obtain that $\mu_{\infty}(x,+\infty) x^{\gamma} \rightarrow 1 /\left(\theta^{\gamma} \gamma \Gamma(\gamma)\right)$ and hence $\mu_{\infty}$ attract the all the initial condition such that $c_{0}^{-}=0$ and $c_{0}^{+}=\theta^{\gamma} /(\gamma \Gamma(\gamma))$.

Proof of $(b)$. Let $X_{1}, \ldots, X_{4}$ be independent random variable with InvGamma $(\gamma, \theta)$ distribution. By (26), $X_{1}+X_{2}$ and $X_{3}+X_{4}$ have density (28), moreover, by (27), $\left(4 B_{1}\right)^{-1}\left(X_{1}+X_{2}\right)$ have the same law of $X_{1}$ and $\left(4 B_{2}\right)^{-1}\left(X_{3}+X_{4}\right)$ have the same law of $X_{3}$, so that $\left(4 B_{1}\right)^{-1}\left(X_{1}+X_{2}\right)+\left(4 B_{2}\right)^{-1}\left(X_{3}+X_{4}\right)$ have the same law of $X_{1}+X_{3}$ which has density (28) again by (26). This show that if $\mu_{\infty}$ has density (26) it is a steady state. Now $\mathbb{E}\left[L^{s}+R^{s}\right]=2 \mathbb{E}\left[L^{s}\right]=g(s)$. So the proof that $\left(H_{0}\right)$ holds is exactly as in part (a). The computation of $\theta$ follows by using Lemma A.1.

\subsection{A further class of explicit solutions}

Let $p, q$ be two numbers in $(0,1)$ such that $p^{\beta}+q^{\beta}=1$ for some $\beta \in(0,1]$ and consider

$$
(L, R)=\left(p U^{\gamma^{*}}, q U^{\gamma^{*}}\right)
$$

with $\gamma^{*}=-(p q)^{\beta} / \beta$ and $U$ uniformly distributed on $(0,1)$. In this case a steady state must satisfy

$$
\hat{\mu}(\xi)=\int_{0}^{1} \hat{\mu}\left(\xi p u^{\gamma^{*}}\right) \hat{\mu}\left(\xi q u^{\gamma^{*}}\right) d u .
$$

Note that, if one looks for positive solutions, the previous equation is still valid when $\hat{\mu}$ is the Laplace transform of $\mu$. Following [11], Sect. 6, let us introduce the change of variable $u^{\gamma^{*}} \xi=y$. Then, standard computations show that equation $(30)$ can be rewritten as

$$
\xi^{1 / \gamma^{*}} \hat{\mu}(\xi)=\frac{1}{\gamma^{*}} \int_{0}^{\xi} y^{1 / \gamma^{*}-1} \hat{\mu}(y p) \hat{\mu}(y q) d y .
$$

Differentiating this equation and rearranging terms, one obtains that $\mu(\cdot)$ satisfies the equivalent differential equation

$$
\gamma^{*} \xi \frac{\partial \hat{\mu}(\xi)}{\partial \xi}=\hat{\mu}(p \xi) \hat{\mu}(q \xi)-\hat{\mu}(\xi) .
$$

Direct computations finally show that

$$
\hat{\mu}(\xi)=\left(1+|\xi|^{\beta}\right) e^{-|\xi|^{\beta}}
$$

solves (31) for all values of $p$ and $q$ satisfying the constraint $p^{\beta}+q^{\beta}=1$, and hence also (30).

When $\beta=1 / 2,(32)$ is the Laplace transform of the inverse Gamma distribution

$$
g_{\infty}(w)=\frac{1}{\Gamma(3 / 2)} \frac{e^{-\frac{1}{w}}}{w^{5 / 2}} \mathbb{I}\{w>0\} .
$$

Using this fact and Lemma A.2, one immediately obtains that if $\beta \in(0,1 / 2]$, then the function $\xi \mapsto \hat{\mu}(\xi)$ defined in (32) is the Laplace transform of a probability distribution $\mu_{\infty}$ on $\mathbb{R}^{+}$while if $\beta \in(1 / 2,1], \xi \mapsto \hat{\mu}(\xi)$ is the Fourier-Stieltjes transform of a (symmetric) probability distribution $\mu_{\infty}$ on $\mathbb{R}$. Moreover, if $\beta=1, \mu_{\infty}$ is explicit, and coincides with the density [4,27]

$$
h_{\infty}(v)=\frac{2}{\pi\left(1+v^{2}\right)^{2}} .
$$


Note that, for $\beta>1$, the function (32) is neither a Fourier-Stieltjes transform, nor a Laplace transform of any probability measure. Indeed, for $\beta>1, \hat{\mu}^{\prime}(0)=\hat{\mu}^{\prime \prime}(0)=0$.

Proposition 3.5. Let $\beta \in(0,1], p \in(0,1)$ and $U$ a random variable with uniform distribution on $(0,1)$. Set $q=\left(1-p^{\beta}\right)^{1 / \beta}, \gamma^{*}=-(p q)^{\beta} / \beta, \alpha=2 \beta$ and $(L, R)=\left(p U^{\gamma^{*}}, q U^{\gamma^{*}}\right)$. Assume that $\left(H_{\alpha}\right)$ holds with $c_{0}^{-}=0$ if $\alpha \in(0,1), c_{0}^{+}=c_{0}^{-}=0$ and $k_{0}>0$ if $\alpha=1$, and $c_{0}^{+}=c_{0}^{-}$if $\alpha \in(1,2]$.

(a) If $0<\beta \leq 1 / 2, \mu(t)$ converges weakly to a stationary state $\mu_{\infty}$ with Laplace transform

$$
\left(1+\frac{\lambda}{2} \xi^{\beta}\right) e^{-\frac{\lambda}{2} \xi^{\beta}} \quad \xi \geq 0
$$

for $\lambda=c_{0}^{+} \pi /(2 \Gamma(\alpha) \sin (\pi \alpha / 2) \cos (\pi \alpha / 2))$ if $\alpha \in(0,1)$ and $\lambda=k_{0}$ if $\alpha=1$.

(b) If $\beta \in(1 / 2,1], \mu(t)$ converges weakly to a stationary state $\mu_{\infty}$ with Fourier-Stieltjes transform

$$
\left(1+\frac{\lambda}{2}|\xi|^{\beta}\right) e^{-\frac{\lambda}{2}|\xi|^{\beta}} \quad \xi \in \mathbb{R}
$$

$$
\text { for } \lambda=c_{0}^{+} \pi /\left(\Gamma(\alpha) \sin (\pi \alpha / 2) \text { if } \alpha \in(1,2) \text { and } \lambda=\sigma_{0}^{2} / 2 \text { if } \alpha=2 .\right.
$$

Proof. Again the proof follows by an application of Theorems 2.2 and 2.3. Note that $\mathbb{E}\left[L^{\alpha}+R^{\alpha}\right]=$ $\left(p^{\alpha}+q^{\alpha}\right) /\left(\alpha \gamma^{*}+1\right)=1$ since $\alpha \gamma^{*}+1=1-2 p^{\beta} q^{\beta}=p^{2 \beta}+q^{2 \beta}=p^{\alpha}+q^{\alpha}$. Moreover, $\mathbb{E}\left[L^{s}+R^{s}\right]=h_{\beta}(s)$ with

$$
h_{\beta}(s)=\frac{p^{s}+q^{s}}{1-s \frac{p^{\beta} q^{\beta}}{\beta}} .
$$

and one can prove that the function $s \mapsto h_{\beta}(s)$ is decreasing in $s=\alpha=2 \beta$ (see Lemma 3.3 in [26]). This shows that $\left(H_{0}\right)$ holds. The explicit evaluation of the constants $\lambda$ follows by using Theorem 2.1.

\section{Applications}

\subsection{Wealth distribution by pure gambling}

As briefly discussed in the introduction, one of the main applications of kinetic models with interactions of type (1) is the mathematical modelling of wealth distribution in a multiagent society in which agents interact by means of binary trades. Among other more realistic models, the pure gambling process [16] describes how wealth distributes for large-enough times when the entire sum of wealths of two agents is up for gambling in each trade and some random fraction of this sum is assigned to one agent and the rest goes to the other. Variants of the original model in [16] have been considered in [8]. Despite its simplicity, the model of pure gambling is able to produce stationary distributions with very different characteristics, which simply follow from the microscopic binary interaction considered for the game.

In [16] the randomness is introduced into the model through a parameter $\varepsilon$ which is a random number taking values in $[0,1]$. The interaction rule reads

$$
v^{\prime}=\varepsilon(v+w), \quad w^{\prime}=(1-\varepsilon)(v+w) .
$$

Some exact steady solutions to the pure gambling model have been presented in [8]. Previous attempts, both from a numerical point of view [21], and theoretical ones [28,30], were in fact able to describe the case in which $\varepsilon$ is a random number with uniform distribution on $(0,1)$. In this case the individual wealth distribution at equilibrium emerges out to be the exponential distribution.

The main feature of trade (37) is that the total amount of wealth which is paid back in a single trade coincides with the amount of wealth the two agents use for gambling (pointwise conservative trade). In other words, in this gambling market agents do not take advantage from the amount of wealth available in the market. In [8] 
the idea of using this wealth as a reservoir for trades has been implemented by allowing agents to trade with random profit

$$
v^{\prime}=\varepsilon_{1}(v+w), \quad w^{\prime}=\varepsilon_{2}(v+w) .
$$

where the parameters $\left(\varepsilon_{1}, \varepsilon_{2}\right)$ are now random numbers drawn from a joint probability distribution such that

$$
\mathbb{E}\left(\varepsilon_{1}+\varepsilon_{2}\right)=1
$$

Within this picture, $v^{\prime}+w^{\prime}$ can be strictly less than $v+w$, and in this case the lost wealth is achieved by the market, or the reverse situation is verified, and the additional wealth is taken from the market. Condition (39), however, guarantees that in the mean the wealth present in the market is left unchanged. This model has been introduced in [8] for a better understanding of the (eventual) formation of tails in the steady distribution. Note that if one assumes that $\varepsilon_{i} \geq \gamma>0, i=1,2$ then agents are prevented from loosing all their wealth in a single trade. Note also that, choosing $\left(\varepsilon_{1}, \varepsilon_{2}\right)=(\varepsilon, 1-\varepsilon)$ the collision rule (38) becomes (37).

\subsubsection{Pure gambling with one lottery}

The pointwise conservative interaction studied in [8] corresponds to the choice $(37)$ with $\varepsilon \sim \operatorname{Beta}(\gamma, \gamma)$. In this case, as already noted, $v^{\prime}+w^{\prime}=v+w$. The corresponding kinetic equation follows in case (a) of Proposition 3.2 with $\alpha=1$ and $(L, R)=(\varepsilon, \varepsilon)$. Consequently the steady states are Gamma distributions of parameter $\gamma$. The particular case in which $\varepsilon$ has uniform distribution on $(0,1)$, is included. In this case $\gamma=1$ and the steady distribution is a exponential distribution.

\subsubsection{Pure gambling with two lotteries}

A different situation appears when the outcome of the trade depends on two independent random results. The outcome in this case is given by (38) where $\varepsilon_{i} \geq 0$ are independent and identically distributed, with $\mathbb{E}\left[\varepsilon_{i}\right]=1 / 2$. By choosing $\varepsilon_{i} \sim \operatorname{Beta}(\gamma, \gamma)$ the corresponding kinetic equation reduces to the case (a) of Proposition 3.2 with $\alpha=1$ and $(L, R)=\left(\varepsilon_{1}, \varepsilon_{1}\right)$. As before, the steady states are given by Gamma distributions of parameter $\gamma$.

The main novelty is represented here by the choice $\varepsilon_{i}=\left(4 B_{i}\right)^{-1}$ with $B_{i} \sim \operatorname{Beta}(\gamma+1 / 2, \gamma-1 / 2)(\gamma>1)$. Then, the kinetic equation corresponds to the case (a) of Proposition 3.4 with $\alpha=1$ and $(L, R)=\left(\varepsilon_{1}, \varepsilon_{1}\right)$. In this case the steady states are inverse-Gamma distributions and possess heavy tails at infinity.

\subsubsection{Mixed gambling with two lotteries}

A third case, which has not been considered in [8], is given by a trade in which the outcome depends on two independent random results, like in the previous case, but the random numbers are mixed in the result. The trade reads

$$
v^{\prime}=\varepsilon_{1} v+\varepsilon_{2} w \quad w^{\prime}=\varepsilon_{2} v+\varepsilon_{1} w .
$$

In (40) the $\varepsilon_{i} \geq 0$ are independent and identically distributed in the interval $(0,1)$, and such that $\mathbb{E}\left[\varepsilon_{1}+\varepsilon_{2}\right]=1$. Also this collision rule is only conservative in mean.

By choosing $\varepsilon_{1} \sim \operatorname{Beta}\left(a_{1}, a_{2}\right)$ and $\varepsilon_{1} \sim \operatorname{Beta}\left(a_{2}, a_{1}\right)$ the corresponding kinetic equation reduces to the case (a) of Proposition 3.2 with $\alpha=1$ and $(L, R)=\left(\varepsilon_{1}, \varepsilon_{2}\right)$. Once again the steady states are Gamma distributions of parameter $a_{1}+a_{2}$. If now $\varepsilon_{i}=\left(4 B_{i}\right)^{-1}$ with $B_{i} \sim \operatorname{Beta}(\gamma+1 / 2, \gamma-1 / 2)(\gamma>1)$, the kinetic equation corresponds to the case (a) of Proposition 3.4 with $\alpha=1$ and $(L, R)=\left(\varepsilon_{1}, \varepsilon_{2}\right)$. Consequently, the steady states are mixture of inverse-Gamma distributions with density given by (28).

\subsection{Dissipative interactions with random fluctuations}

In 2003 Ben-Avraham and coworkers [9] introduced a one-dimensional model of the Boltzmann equation, in which binary collision processes are given by a collision rule of type (1), where $p_{1}=q_{2}=p$ and $p_{2}=q_{1}=q$, with $p$ and $q$ positive constants. Hence

$$
v^{\prime}=p v+q w \quad w^{\prime}=q v+p w
$$


As observed in [9], while in the long-time limit velocity distributions are generically self-similar, there is a wide spectrum of possible behaviors for the solution of the kinetic equation. The velocity distributions are characterized by algebraic or stretched exponential tails and the corresponding exponents depend sensitively on the collision parameters. Interestingly, when there is energy or momentum conservation, the behavior is universal. Momentum conservation corresponds to the choice

$$
p+q=1 .
$$

In this case, the bilinear kinetic equation represents the simplest one-dimensional model of a dissipative gas. Indeed, energy is dissipated in any single collision, since

$$
v^{\prime 2}+w^{\prime 2}=v^{2}+w^{2}-2 p q(v-w)^{2} .
$$

In classical kinetic theory of rarefied gases, dissipation is usually expressed in terms of the so-called coefficient of restitution, which relates the absolute values of the relative velocities before and after collisions. A dissipative collision is such that momentum is preserved, while

$$
\left|v^{\prime}-w^{\prime}\right|=e|v-w|,
$$

with $0<e<1$. In collisions of type (41), in case (42) holds, one has $e=|p-q|<1$. Note that collisions of type (41) also include the case of sticky particles, which correspond to $p=q=1 / 2$. In this case, in fact, the coefficient of restitution $e=0$, and

$$
v^{\prime}=w^{\prime}=\frac{1}{2}(v+w) .
$$

Suppose now that the coefficient of restitution is a random variable, in the form $\tilde{e}=\vartheta e$, where $\mathbb{E}\left(\vartheta^{2}\right)>1$. Then, $\mathbb{E}\left(\tilde{e}^{2}\right)=e^{2} \mathbb{E}\left(\vartheta^{2}\right)>e^{2}$. Note that this choice corresponds to change the mixing parameters $p$ and $q$ into $\tilde{p}=\vartheta p$, and $\tilde{q}=\vartheta q$.

Let us choose the random variable $\vartheta$ in such a way that $\mathbb{E}(\vartheta)>1$, and at the same time $\mathbb{E}\left(\vartheta^{2}\right)\left(p^{2}+q^{2}\right)=1$. Then, in the case in which the initial mean value of the solution of the kinetic equation (4) is assumed equal to zero, so that it remains equal to zero at subsequent times, the second moment of the solution to (4) is preserved (provided that it is finite at time 0). This idea has been developed in [14] for the three-dimensional dissipative Boltzmann equation for Maxwell molecules introduced in [10]. The choice of a random coefficient of restitution was there motivated by the presence of an external background, able to generate random fluctuations in any collision. The analysis of [14] put in evidence that conservation of energy in the mean is responsible of the development of equilibria with heavy tails. However, it was impossible to obtain examples of explicit equilibria.

In the one-dimensional setting, the binary collision proposed in [14] can be replaced by

$$
v^{\prime}=\vartheta(p v+q w) \quad w^{\prime}=\vartheta(q v+p w) .
$$

where $p, q, \vartheta$ satisfy

$$
p+q=1, \quad \mathbb{E}(\vartheta)>1, \quad \mathbb{E}\left(\vartheta^{2}\right)\left(p^{2}+q^{2}\right)=1 .
$$

Collisions of type (45) describe a toy model in which the result of the binary collision depends on the simultaneous action of two different effects. The first, described by the collision in which $\theta$ is assumed equal to one, represents a simple dissipative collision. This collision is then changed by a multiplicative random perturbation (the multiplication by $\theta$ ), which can be seen as the effect of the environment. The combined effect of dissipation and random perturbation is such that the energy is preserved in the mean, due to the second condition in (46) and the assumption of zero mean value. Thanks to Theorem 2.2, if conditions (46) are satisfied, the solution to (4) converges towards a steady state $\mu_{\infty}(v)$. In reason of Proposition 3.5, by choosing $\vartheta=U^{-p q}$, where $U$ is a uniform variable on the interval $(0,1), \vartheta$ satisfies conditions $(46)$, and the steady state $\mu_{\infty}(\xi)$ now takes the form (36), with $\lambda=\sigma_{0}^{2} / 2$ and $\beta=1$. As discussed in Section 3.4, in this case the steady state has an explicit form in the physical space, given by (34), and possesses heavy tails at infinity. 
In the present one-dimensional situation, analogous reasoning leads to consider, for $1 / 2<\beta \leq 1$ mixing parameters $p$ and $q$ such that $p^{\beta}+q^{\beta}=1$, and random mixing parameters $\tilde{p}$ and $\tilde{q}$ which are obtained from $p$ and $q$ by multiplying them by a random variable $\vartheta=U \gamma^{*}$, where $\gamma^{*}=-(p q)^{\beta} / \beta$, where as before $U$ is a uniform distribution on the interval $(0,1)$. Then, the result of Proposition 3.5 insures that the kinetic model has a solution which converges to an explicit steady state as given by its Fourier transform (36).

\section{SELF-Similar SOLUTIONS}

The analysis of the previous Section can be easily adapted to obtain some explicit self-similar solutions of bilinear models of type (4). Self-similar solutions can appear in (4) as soon as conservation laws in the binary collisions are missing. Two cases have been recently discussed, which are linked respectively to dissipative kinetic theory [4] and to kinetic modelling of nonconservative economies [31]. In the former case, an explicit solution of type (34) has been obtained in [4] as self-similar solution of a one-dimensional kinetic model of type (4), modelling binary dissipative interactions. In this case, while there is conservation of momentum, the second moment is exponentially decaying to zero (cooling effect). In the latter case, an analytical solution of type (23) with $\gamma=3 / 2$ has been discovered in [31] as self-similar solution of a kinetic model for wealth distribution, in which the mean wealth is not preserved. Motivated by the analogy with a dissipative Maxwell gas, Slanina [31] considered a bilinear kinetic model of Boltzmann type, based on dissipative binary collisions (41) with $\sqrt{p}+\sqrt{q}=1$ and $p, q \in(0,1)$. In this case $v^{\prime}+w^{\prime}=(1-2 \sqrt{p q})(v+w)<v+w$, which implies that the mean value decays exponentially to zero, with $m(t)=\int v \mu(t, d v)=m(0) e^{-2 \sqrt{p q} t}$.

The standard way to look for the existence of self-similar solution is to consider a scaling of the solution to (4) in such a way that the missing conservations are restored. For example, in the wealth case considered by Slanina, after scaling the new solution has to be such that its mean value remains constant. This can be easily done through the scaling (in Fourier variables) $\hat{g}(t, \xi)=\hat{\mu}(t, \xi / m(t))$. Indeed, this scaling implies that $\int v g(t, d v)=1$ for all $t \geq 0$.

This procedure can be applied in more generality. Let us assume that $p^{\beta}+q^{\beta}=1$, for some $\beta \in(0,1]$. The scaling of the solution (in Fourier variables) in this case reads

$$
\hat{g}(t, \xi)=\hat{\mu}\left(t, \xi e^{-\gamma^{*} t}\right)
$$

where $\gamma^{*}=-(p q)^{\beta} / \beta$. It is standard to verify that the equation satisfied by $\hat{g}$ takes the form

$$
\frac{\partial \hat{g}(t, \xi)}{\partial t}+\xi \gamma^{*} \frac{\partial \hat{g}(t, \xi)}{\partial \xi}=\hat{g}(t, p \xi) \hat{g}(t, q \xi)-\hat{g}(t, \xi)
$$

Using the results of [5] (see also [11]), one can prove that if $\mu_{0}$ satisfies $\left(H_{\alpha}\right)$ for $\alpha=2 \beta$, then $g(t, \cdot)$ converges (weakly) to a steady state. Moreover, the steady solutions to equation (47) solve the equation

$$
\xi \gamma^{*} \frac{\partial \hat{g}(\xi)}{\partial \xi}=\hat{g}(p \xi) \hat{g}(q \xi)-\hat{g}(\xi)
$$

which is equivalent, as shown in Section 3.4, to (30). The two relevant cases studied in [4] and [31] correspond to $\beta=1$ and $\beta=1 / 2$, respectively. In both cases, the solutions are explicit in the physical space. If $\beta=1$, the steady state which generates the self-similar solution by reverting to the original density is given by (34). If $\beta=1 / 2$ the steady state is given by (23) with $\gamma=3 / 2$.

Thanks to the equivalence between (47) and (30), all the other cases for $\beta$ can be treated as well, to obtain selfsimilar solutions with an explicit description in Fourier or Laplace variables. Unfortunately, to these solutions do not correspond explicit solutions in the physical space. 


\section{Conclusions}

Bilinear kinetic models of Maxwell-Boltzmann type have a wide range of applications. By virtue of the possibility to consider a variety of binary interactions, these models can be fruitfully applied to different multiagent systems, ranging from economics to kinetic theory of dissipative gases. In most cases, the explicit form of the steady states and of the self-similar solutions is unknown, and one can only access few properties, including fat tails at infinity and/or existence of a limited number of moments. In some cases, however, the particular form of the binary interaction allows to recover explicitly stationary solutions and self-similar solutions. In this note, we reviewed briefly all the known cases in which solutions can be made explicit, and we added an almost complete description of the underlying binary interactions.

\section{A. Appendix}

Lemma A.1. For $i=1,2$, let $X_{i} \sim \operatorname{Inv} \operatorname{Gamma}(\gamma, \theta)$ independent random variables. Then

$$
\lim _{x \rightarrow+\infty} x^{\gamma} P\left\{X_{1}>x\right\}=\frac{\theta^{\gamma}}{\gamma \Gamma(\gamma)} .
$$

and

$$
\lim _{x \rightarrow+\infty} x^{\gamma} P\left\{X_{1}+X_{2}>x\right\}=\frac{2 \theta^{\gamma}}{\gamma \Gamma(\gamma)} .
$$

Proof. If $X$ is $\operatorname{Inv} \operatorname{Gamma}(\gamma, \theta)$ then $1 / X \sim \operatorname{Gamma}(\gamma, 1 / \theta)$, hence

$$
x^{\gamma} P\{X>x\}=\frac{\theta^{\gamma} x^{\gamma}}{\Gamma(\gamma)} \int_{0}^{\frac{1}{x}} y^{\gamma-1} e^{-y \theta} d y=\frac{\theta^{\gamma}}{\Gamma(\gamma)} u^{\gamma} \int_{0}^{1 / u} y^{\gamma-1} e^{-y} d y
$$

where $u=x / \theta$. Using the fact that $e^{-y}=\sum_{k \geq 0}(-y)^{k} / k$ ! and integrating term by term the first part of the thesis follows. The second part is an easy consequence of the first part and of well-known facts about the tail behavior of the convolution of two distribution functions. Indeed if $1-F_{i}(x) \sim c_{i} x^{-\gamma}$ for $i=1,2$, then $1-F_{1} * F_{2}(x) \sim\left(c_{1}+c_{2}\right) x^{-\gamma}$ (cf. pg. 271 of [19]).

Lemma A.2. Let $L(\xi)$ be the Laplace transform of a positive random variable. If $\alpha \in(0,1)$ then $L\left(\xi^{\alpha}\right),(\xi>0)$ is the Laplace transform of a probability distribution $\mu_{\infty}$ on $\mathbb{R}^{+}$. Moreover, if $\alpha \in(0,2], \xi \mapsto L\left(|\xi|^{\alpha}\right)$ is the Fourier-Stieltjes transform of a (symmetric) probability distribution $\mu_{\infty}$ on $\mathbb{R}$.

Proof. Let $\alpha<1$. Let $X$ be a (positive) random variable with Laplace transform $L$ and $S_{\alpha}$ a (positive) stable random variable with Laplace transform $\mathbb{E}\left[\exp \left\{-\xi S_{\alpha}\right\}\right]=\exp \left\{-\xi^{\alpha}\right\}(\xi>0)$. When $S_{\alpha}$ and $X$ are independent, it is easy to check that the Laplace transform of $X^{1 / \alpha} S_{\alpha}$ is given by $L\left(\xi^{\alpha}\right)$. Analogously if $\alpha \in(0,2]$, introduce the (symmetric) stable random variable $S_{\alpha}$ with characteristic function $\mathbb{E}\left[\exp \left\{i \xi S_{\alpha}\right\}\right]=\exp \left\{-|\xi|^{\alpha}\right\}$. The characteristic function of $X^{1 / \alpha} S_{\alpha}$ is $L\left(|\xi|^{\alpha}\right)$.

\section{REFERENCES}

[1] J. Aaronson and M. Denker. Characteristic functions of random variables attracted to 1-stable laws. Ann. Probab., 26(1):399$415,1998$.

[2] G. Alsmeyer, J.D. Biggins, and M. Meiners. The functional equation of the smoothing transform. Ann. Probab., 40(5):2069$2105,2012$.

[3] G. Alsmeyer and M. Meiners. Fixed points of the smoothing transform: two-sided solutions. Probab. Theory Related Fields, 155(1-2):165-199, 2013.

[4] A. Baldassarri, A. Puglisi, and U. Marini Bettolo Marconi. Kinetics models of inelastic gases. Math. Models Methods Appl. Sci., 12(7):965-983, 2002. Special issue on kinetic theory.

[5] F. Bassetti and L. Ladelli. Self-similar solutions in one-dimensional kinetic models: a probabilistic view. Ann. Appl. Probab., 22(5):1928-1961, 2012. 
[6] F. Bassetti, L. Ladelli, and D. Matthes. Central limit theorem for a class of one-dimensional kinetic equations. Probab. Theory Related Fields, 150(1-2):77-109, 2011.

[7] F. Bassetti and E. Perversi. Speed of convergence to equilibrium in Wasserstein metrics for Kac-like kinetic equations. Electron. J. Probab., 18:no. 6, 35, 2013.

[8] F. Bassetti and G. Toscani. Explicit equilibria in a kinetic model of gambling. Phys. Rev. E (3), 81(6):066115, 7, 2010.

[9] D. Ben-Avraham, E. Ben-Naim, K. Lindenberg, and A. Rosas. Self-similarity in random collision processes. Phys. Rev. E, 68:R050103, 2003.

[10] A.V. Bobylev, J.A. Carrillo, and I.M. Gamba. On some properties of kinetic and hydrodynamic equations for inelastic interactions. J. Statist. Phys., 98(3-4):743-773, 2000.

[11] A.V. Bobylev, C. Cercignani, and I.M. Gamba. On the self-similar asymptotics for generalized nonlinear kinetic Maxwell models. Comm. Math. Phys., 291(3):599-644, 2009.

[12] L. Boudin and F. Salvarani. A kinetic approach to the study of opinion formation. M2AN Math. Model. Numer. Anal., 43(3):507-522, 2009.

[13] L. Boudin and F. Salvarani. Modelling opinion formation by means of kinetic equations. In Mathematical modeling of collective behavior in socio-economic and life sciences, Model. Simul. Sci. Eng. Technol., pages 245-270. Birkhäuser Boston, Inc., Boston, MA, 2010.

[14] J.A. Carrillo, S. Cordier, and G. Toscani. Over-populated tails for conservative-in-the-mean inelastic Maxwell models. Discrete Contin. Dyn. Syst., 24(1):59-81, 2009.

[15] L. Devroye. A note on Linnik's distribution. Statist. Probab. Lett., 9(4):305-306, 1990.

[16] A. Dragulescu and V.M. Yakovenko. Statistical mechanics of money. Eur. Phys. Jour. B, 17:723-729, 2000.

[17] R. Durrett and T.M. Liggett. Fixed points of the smoothing transformation. Z. Wahrsch. Verw. Gebiete, 64(3):275-301, 1983.

[18] A. Erdélyi, W. Magnus, F. Oberhettinger, and F. G. Tricomi. Higher transcendental functions. Vols. I. McGraw-Hill Book Company, Inc., New York-Toronto-London, 1953. Based, in part, on notes left by Harry Bateman.

[19] W. Feller. An introduction to probability theory and its applications. Vol. II. Second edition. John Wiley \& Sons Inc., New York, 1971.

[20] I.S. Gradshteyn and I.M. Ryzhik. Table of integrals, series, and products. Elsevier/Academic Press, Amsterdam, seventh edition, 2007. Translated from the Russian, Translation edited and with a preface by Alan Jeffrey and Daniel Zwillinger, With one CD-ROM (Windows, Macintosh and UNIX).

[21] A.K. Gupta. Models of wealth distributions: a perspective. In Econophysics and sociophysics: trends and perspectives.

[22] I.A. Ibragimov and Yu.V. Linnik. Independent and stationary sequences of random variables. Wolters-Noordhoff Publishing, Groningen, 1971. With a supplementary chapter by I. A. Ibragimov and V. V. Petrov, Translation from the Russian edited by J. F. C. Kingman.

[23] A.M. Iksanov. Elementary fixed points of the BRW smoothing transforms with infinite number of summands. Stochastic Process. Appl., 114(1):27-50, 2004.

[24] J.V. Linnik. Linear forms and statistical criteria. II. In Selected Transl. Math. Statist. and Prob., Vol. 3, pages 41-90. Amer. Math. Soc., Providence, R.I., 1962.

[25] Q. Liu. Fixed points of a generalized smoothing transformation and applications to the branching random walk. Adv. in Appl. Probab., 30(1):85-112, 1998.

[26] L. Pareschi and G. Toscani. Self-similarity and power-like tails in nonconservative kinetic models. J. Stat. Phys., 124(2-4):747$779,2006$.

[27] L. Pareschi and G. Toscani. Interacting multiagent systems. Kinetic equations 8 Monte Carlo methods. Oxford University Press, Oxford, 2013.

[28] M. Patriarca, A. Chackraborti, and K. Kaski. Statistical model with a standard $\gamma$ distribution. Phys. Rev. E, 70:016104, 2004.

[29] C. Radhakrishna Rao. On some problems arising out of discrimination with multiple characters. Sankhyā, 9:343-366, 1949.

[30] P. Repetowicz, S. Hutzler, and P. Richmond. Dynamics of money and income distributions. Physica A, 356:641-654, 2005.

[31] F. Slanina. Inelastically scattering particles and wealth distribution in an open economy. Phys. Rev. E, $69,2004$.

[32] G. Toscani. Kinetic models of opinion formation. Commun. Math. Sci., 4(3):481-496, 2006.

[33] V.M. Zolotarev. One-dimensional stable distributions, volume 65 of Translations of Mathematical Monographs. American Mathematical Society, Providence, RI, 1986. Translated from the Russian by H. H. McFaden, Translation edited by Ben Silver. 